\title{
Birgit Eckardt*
}

\section{,Scheiden tut weh" - \\ Kommunikationsstörungen zwischen Fachleuten und Laien im Ehescheidungsrecht}

\begin{abstract}
Like any other science, the jurisprudence uses a cant, that is not self -explanatory for the layman. It is even more difficult, that in certain legal fields laymen are not only the affected ones, but also the agents, who require the secular intermediation of a solicitor. Thereby occurring communication problems are attributed to the large terminology.

It will be shown by means of a four step communication model while using the example of the divorce legislation, why and on what communication levels the communication between experts and laymen is disturbed. The interdisciplinary research approach, that considers linguistic and jurisprudential as well as psychological and sociological aspects, shows the rather unexpected report, that communication perturbations between solicitors and clients occur to the same extent on the content level as on the relationship level. Objective misunderstandings are easier to remove than problems on the relationship level.

Communication problems between judges and parties occur mainly during the very formalised (written) proceedings because of factual ignorance. The oral proceedings take place mainly undisturbed, when judges and solicitors measure up their role as mediator, respectively conciliator.
\end{abstract}

\section{Untersuchungsgegenstand und methodische Vorüberlegungen}

\subsection{Interdisziplinärer Forschungsansatz}

„Der Zaun, der Germanisten und Juristen in ihrer Arbeit voneinander trennt, ist hoch. Selten wagt es heute ein Gelehrter, sich mit Gegenständen

\footnotetext{
* Birgit Eckardt

Friedrich-Schiller-Universität Jena

Gleichstellungsbüro

Fürstengraben 1

D-07743 Jena
} 
zu beschäftigen, für die eine andere Fachwissenschaft als zuständig gilt. Über diesem Takt, der in Wahrheit oft Feigheit ist, bleiben leicht Forschungsfelder unbearbeitet, auf denen bei gemeinsamer Arbeit viel zu ernten wäre" (Hattenhauer 1995: 17). So treffend beschreibt der Jurist Hans Hattenhauer das Dilemma des interdisziplinären (Nicht)zusammenarbeitens von Sprach-und Rechtswissenschaftlern. Doch Ausnahmen bestätigen die Regel und jeder Gartenzaun verfügt über eine Gartentür, durch die man wechselseitig das jeweilige Nachbargrundstück betreten und sich darin umschauen kann. Die Ergebnisse der nachfolgenden empirischen Untersuchung basieren deshalb auf einem interdisziplinären Forschungsansatz. ${ }^{1}$ Die Rechtsordnung berührt alle Lebensbereiche und hat eine besondere Beziehung zu Kommunikation und Sprache, denn nur mittels Sprache ist es überhaupt möglich, bestimmte Rechtsnormen festzuschreiben. Insbesondere das Familienrecht hat vielfältige Auswirkungen auf das Leben der Menschen. Stärker als in anderen Bereichen tangiert diese Rechtsmaterie den Laien und zwingt ihn zur Auseinandersetzung, die oft nur durch die fachsprachliche Vermittlung des Anwalts erfolgen kann. Der nicht zu unterschätzende Einfluss des Familienrechts auf die Privatsphäre des einzelnen führt dazu, dass sich gerade in diesem Bereich die unterschiedlichen Ebenen, auf denen Kommunikation stattfindet, besonders gut beschreiben und untersuchen lassen. Außerdem unterliegt das Familienrecht durch verschiedene Reformen im Kindschaftsrecht und im Eheschließungsrecht einer Dynamik, die sich auch auf die sprachliche Entwicklung und auf das Kommunikationsverhalten auswirkt.

\subsection{Sprachwissenschaftliche Grundlagen}

Jeder Sprachbenutzer ist schon einmal in der Situation gewesen, dass er auf einem Gebiet, wo er Laie ist, kein richtiges Gespräch führen konnte, weil ihm der jeweilige Fachjargon nicht geläufig war. Gerade die juristische Fachsprache gilt nicht nur für den Laien, sondern auch für den Sprachwissenschaftler als schwer verständlich, weil sie über einen ständig anwachsenden Fachwortschatz verfügt, der zur Kommunikations-

1 Dabei kommt der Autorin zugute, dass sie als Sprachwissenschaftlerin auch Rechtswissenschaften im Nebenfach studiert hat. 
barriere wird. Kommunikationsbarrieren treten immer dann auf, ,wenn Fachsprachen in die gesamtgesellschaftliche Diskussion einfließen, ihr Wissen aber nicht allgemein vermitteln " (Fluck 1996: 39). Und genau diese Konstellation findet sich in der kommunikativen Anwendung der juristischen Fachsprache, zum Beispiel im Gespräch zwischen dem Anwalt und seinen Mandanten oder in der mündlichen Gerichtsverhandlung. Doch nicht nur der umfangreiche Fachwortschatz führt zu Verständigungsschwierigkeiten.

Traditionell beschäftigte sich die Fachsprachenforschung mit morphologischen, syntaktischen und lexiko-semantischen Aspekten des schriftlichen Fachsprachengebrauchs (Selting 1987: 20); doch es wird zunehmend eine Ausweitung des Forschungsgegenstandes gefordert: ,Hiermit tritt die Untersuchung mündlicher Kommunikation wie auch die Beschäftigung mit Verständigungsprozessen in mündlicher und schriftlicher Kommunikation in den Vordergrund. Gemäß der Unterscheidung zwischen fachinterner und fachexterner Kommunikation wird die Berücksichtigung des sozialen Kontextes bei der Analyse und Kritikfachsprachlicher Texte hinsichtlich ihrer 'Schwerverständlichkeit, verlangt." (Selting 1987: 20). Doch wodurch wird diese Schwerverständlichkeit der juristischen Fachsprache hervorgerufen? Ist es wirklich nur der Fachwortschatz, der nicht allgemeinverständlich ist?

Die empirische Untersuchung der Gesprächskonstellation Anwalt/ Mandant und Richter/Mandant+ Anwalt basiert neben soziolinguistischen Erkenntnissen auf dem modifizierten Kommunikationsmodell von Friedemann Schulz von Thun. Nach diesem Modell findet Kommunikation auf vier verschiedenen Ebenen statt, wie das Nachrichtenquadrat (Schulz von Thun 1981: 44 ff.) verdeutlicht:

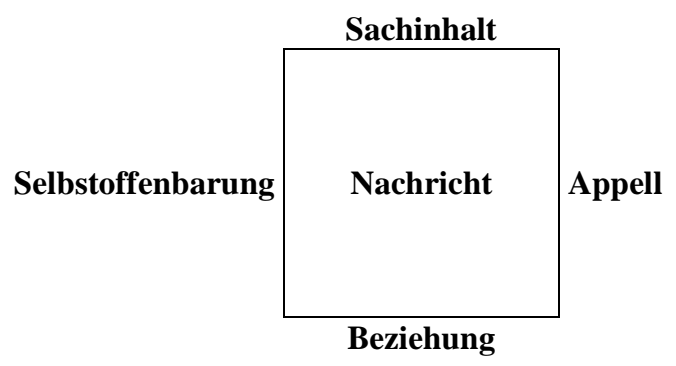


Ein und dieselbe Nachricht enthält demzufolge mehrere Botschaften auf vier verschiedenen Ebenen:

\begin{tabular}{|c|c|}
\hline Sachinhaltsebene & Worüber informiere ich? \\
\hline Selbstoffenbarungsebene & Was gebe ich von mir kund? \\
\hline Beziehungsebene & $\begin{array}{c}\text { Was halte ich von meinem } \\
\text { Kommikationspartner und wie stehen wir } \\
\text { zueinander? }\end{array}$ \\
\hline Appellebene & $\begin{array}{c}\text { Wozu möchte ich meinen } \\
\text { Kommunikationspartner veranlassen? }\end{array}$ \\
\hline
\end{tabular}

Tabelle 1

Ausgehend von einem bi-direktionalen Verlauf der Kommunikation, wie ihn die Dialog-Situation zwischen Anwalt und Mandanten vorgibt, müssen sich beide Kommunikationspartner auf allen vier Ebenen bewegen können. Dominiert bei einem der beiden Kommunikationsteilnehmer nur eine der vier Ebenen, kann das bereits zu Verständigungsschwierigkeiten führen. Dabei kann der Sender einer Nachricht auswählen, auf welche Kommunikationsebene er seinen Schwerpunkt legt; der Empfänger der Nachricht muss grundsätzlich auf allen vier Ebenen hören, weshalb Schulz von Thun auch vom ,vierohrigen Empfänger" (Schulz von Thun 1981: 44) spricht. Bezüglich seiner Reaktion hat der Nachrichtenempfänger dann wiederum die freie Auswahl, auf welche der vier Ebenen der Nachricht er reagieren will. Diese freie Auswahl führt dann zu Kommunikationsproblemen, ,, wenn der Empfänger auf eine Seite Bezug nimmt, auf die der Sender das Gewicht nicht legen wollte. Oder wenn der Empfänger überwiegend nur mit einem Ohr hört, und damit taub ist (oder sich taub stellt) für alle Botschaften, die sonst noch ankom-

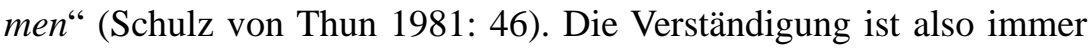
dann gestört, wenn gesendete und empfangene Nachricht nicht übereinstimmen. Erschwert wird der Kommunikationsprozess auch dadurch, dass die Abgrenzung der einzelnen Kommunikationsebenen sehr kompliziert und nicht eindeutig ist, da sie nicht streng voneinander getrennt existieren, 
sondern sich wechselseitig beeinflussen und durchdringen. Weitere sprachliche und außersprachliche Faktoren wie zum Beispiel die Kommunikationssituation, die wechselseitigen Rollenerwartungen oder die aufgrund unterschiedlicher individuell geprägter Erfahrung gebildeten Begrifflichkeiten bestimmen ebenfalls die Kommunikation und müssen berücksichtigt werden.

\section{Ergebnisse der empirischen Untersuchung}

\subsection{Rechtliches Korpus und sprachwissenschaftliche Methodik}

Anhand ausgewählter Ergebnisse der empirischen Untersuchung soll das bisher Gesagte präzisiert werden. ${ }^{2}$ Die Gesamtuntersuchung bezieht sich auf zwei Problemkomplexe: im ersten Teil wurden Kommunikationsstörungen zwischen Anwalt und Mandanten analysiert, der zweite Teil beschäftigte sich mit Kommunikationsstörungen, die im gerichtlichen Verfahren zwischen Richter und Parteien auftreten. Im nachfolgenden soll die Kommunikation zwischen Anwalt und Mandanten analysiert werden.

Dazu wurden Rechtsanwälte aus 15 Anwaltskanzleien der alten und neuen Bundesländer befragt. Mit ihrer Hilfe wurden anhand von Fragebögen 55 Scheidungsfälle aus der anwaltlichen Praxis auf Kommunikationsstörungen untersucht. Die Auswertung der Fragebögen wurde durch persönliche Interviews mit Anwälten und betroffenen Scheidungsparteien sowie durch Teilnahme der Autorin an Ehescheidungsverhandlungen ergänzt.

Basis der Fragebögen ist das bereits vorgestellte Kommunikationsmodell unter Einbeziehung von soziologischen und psychologischen Aspekten. Das Kommunikationsmodell ist wiederum mit einem juristischen Raster verknüpft, das Anwälte bei der Vorbereitung einer Ehescheidung verwenden. ${ }^{3}$

\footnotetext{
2 Die Gesamtergebnisse der Untersuchung sind nachzulesen in: Eckardt, Birgit (2000). Fachsprache als Kommunikationsbarriere. Verständigungsprobleme zwischen Juristen und Laien. Wiesbaden: Deutscher Universitäts-Verlag GmbH.

3 Dabei wird unterschieden nach Scheidungsvoraussetzungen, die erfüllt sein müssen, um einen Scheidungsantrag bei Gericht einreichen zu können sowie Scheidungsfolgen 1 und 2. Scheidungsfolgen 1 müssen von Amts wegen geregelt werden; Scheidungsfolgen 2 sind fakultativ.
} 
Bei der Auswertung werden auch Aspekte des soziolinguistischen Varietätenmodells von Löffler (Löffler 1994: 86) sowie Erkenntnisse der sprachlichen Charakterisierung der juristischen Fachsprache berücksichtigt.

\subsection{Soziologische Angaben}

\begin{tabular}{|c|c|c|}
\hline Geschlecht & $\begin{array}{c}\text { männlich } \\
\text { weiblich }\end{array}$ & $\begin{array}{l}60 \% \\
40 \%\end{array}$ \\
\hline Alter & $\begin{array}{c}\text { 18-30 Jahre } \\
\text { 31-45 Jahre } \\
\text { 46-60 Jahre } \\
\text { über } 60 \text { Jahre }\end{array}$ & $\begin{array}{l}18 \% \\
55 \% \\
27 \% \\
---\end{array}$ \\
\hline Ausbildung & $\begin{array}{l}\text { Lehre } \\
\text { Fachschulausbildung } \\
\text { Hochschulausbildung }\end{array}$ & $\begin{array}{l}42 \% \\
27 \% \\
31 \%\end{array}$ \\
\hline Beruf & $\begin{array}{c}\text { angestellt } \\
\text { selbständig } \\
\text { nicht berufstätig }\end{array}$ & $\begin{array}{l}60 \% \\
16 \% \\
24 \%\end{array}$ \\
\hline Herkunft & $\begin{array}{c}\text { Neue Bundesländer } \\
\text { Alte Bundesländer } \\
\text { Ausländer }\end{array}$ & $\begin{array}{r}73 \% \\
18 \% \\
9 \%\end{array}$ \\
\hline
\end{tabular}

Tabelle 2

Tabelle 2 enthält einige wichtige Angaben zur Mandantenstruktur, die interessante Gliederungshilfen für die einzelnen Fallgruppen sind. ${ }^{4}$ Obwohl bei den untersuchten Fällen die Anzahl der männlichen Scheidungsantragsteller überwiegt, entspricht das nicht dem bundesdeutschen Durchschnitt, nachdem bereits Mitte der achtziger Jahre mehr als zwei Drittel der Scheidungsanträge von Frauen eingereicht wurden. Diese Abweichung kommt dadurch zustande, dass die von den Anwälten ausgesuchten Fälle sich nicht primär an geschlechterspezifischen Aspekten orientierten, sondern unter dem Aspekt ausgewählt wurden, dass die Fälle

4 Das Zahlenmaterial der Tabellen 2-5 bezieht sich auf die in den Fragebögen ausgewerteten 55 Scheidungsfälle. 
stellvertretend für unterschiedliche Kommunikationskonstellationen stehen sollten. Die Juristen bestätigten jedoch, dass tendenziell mehr Frauen als Männer Scheidungsanträge stellen, wobei die Quote naturgemäß von Anwalt zu Anwalt schwankt.

Die altersspezifische Verteilung zeigt, dass immer mehr Ehen nach verhältnismäßig kurzer Dauer (also in einem mittleren Altersbereich zwischen 31-45 Jahren) geschieden werden. Weiterhin gibt es eine Scheidungshäufung bei Langzeitehen, wenn eine Neuorientierung der Partner ohne die mittlerweile erwachsenen Kinder erfolgt.

Bezüglich des (Aus)bildungsstandes der Antragsteller bietet sich ein stark differenziertes Bild: Sowohl ein eher niedriges Bildungsniveau als überraschenderweise auch ein hohes Bildungsniveau geben Anlass zu Kommunikationsstörungen. Antragsteller mit höherem Bildungsniveau verfügen oft über juristisches Halbwissen, das die Kommunikation zwischen Anwalt und Mandanten erschwert. In diesem Zusammenhang spielen auch die Erwartungshaltung und das Rollenverständnis eine große Rolle. So ist die Erwartungshaltung von Antragstellern mit eher niedrigem Bildungsstand an den Anwalt meist sehr hoch. Sie haben geradezu Ehrfurcht vor dem Fachmann und vertrauen ihm blind. Dagegen steigt die Skepsis gegenüber dem Anwalt mit der Zunahme des Bildungsniveaus. Aussagen des Prozessvertreters werden kritisch, zum Teil auch misstrauisch, hinterfragt. Es ist aber auch die gegenläufige Tendenz zu beobachten, dass Antragsteller aufgrund mangelnden Wissens das Handeln des Anwalts nicht einschätzen können und ihm deshalb als „Geldabschneider" misstrauen. Dagegen vertrauen viele Antragsteller mit höherem Bildungsniveau der umfassenden Ausbildung des Juristen, und suchen sich gezielt einen Spezialisten für ihren Fall.

Wichtig für den kommunikativen Kontext ist auch die berufliche Position des Antragstellers. So treten einige Kommunikationsstörungen zum Beispiel bei Selbständigen kaum auf, weil wesentliche Streitpunkte bereits vor der Scheidungsklage mittels Ehevertrag unstreitig geklärt wurden. Probleme gibt es auch, wenn ein Ehepartner durch seine berufliche Tätigkeit sehr prominent ist und im Blickpunkt des öffentlichen Interesses steht. Diese Prominenz kann zu einem medienwirksamen Scheidungskrieg genutzt werden und zwar nicht nur vom prominenten Teil, sondern auch von dessen Ehepartner: Durch einen solchen Schritt wird eine sachliche Kommunikation fast immer vereitelt, denn gerade die Sensations- 
presse spielt mit den unterschiedlichen Emotionen und verfälscht die Sachlage durch Pauschalisierungen.

Ob die Mandanten aus den neuen oder aus den alten Bundesländern kommen, ist für die Kommunikation beim Anwalt nur von marginaler Bedeutung. Problematischer ist es dagegen, wenn der Antragsteller Ausländer ist. Dabei steht nicht - wie eigentlich erwartet - die mangelnde Sprachkompetenz als Kommunikationshindernis im Vordergrund, sondern eher mentalitätsgebundene oder/ und religiöse Eheverständnisse. So bestand bei einer Ehescheidung nach türkischem Recht das Problem, dass der Mann als Antragsteller nicht einsah, dass seine Frau die Entscheidung über die Ehescheidung mittragen muss und eine ,einseitige“ Scheidung nur unter sehr engen Voraussetzungen möglich ist. Bei einem Antragsteller aus Süd-Korea lag die Schwierigkeit darin, dass dieser Mann aufgrund seiner Mentalität nicht in der Lage war, Aussagen auf der Selbstoffenbarungsebene zu treffen, um sein Anliegen sowie die Beweggründe für seinen Antrag darzulegen.

\subsection{Scheidungsvoraussetzungen}

Bei den Scheidungsvoraussetzungen wird unterschieden zwischen der einverständlichen Scheidung, der streitigen Scheidung sowie der Härtescheidung.

Über 60 Prozent der Scheidungen werden einverständlich beantragt. Schon dieser Fachterminus führt häufig zu Missverständnissen auf der Sachinhaltsebene, denn einverständlich heißt eben nicht, dass man in völliger Harmonie und Übereinstimmung auseinandergeht, sondern einverständlich heißt lediglich, dass beide Ehepartner grundsätzlich der Scheidung zustimmen und sich über die Folgen dieser Entscheidung einigen. 
Tabelle 3

Wie Tabelle 3 zeigt, treten erwartungsgemäß die meisten Kommunikationsstörungen bei streitigen Scheidungen sowie bei Härtescheidungen auf. Bezüglich der Kommunikationsebenen sind vor allem die Sachinhaltsebene, die Beziehungsebene und die Selbstoffenbarungsebene betroffen. Die Appellebene folgt mit geringem Abstand. Da die Abgrenzung der einzelnen Kommunikationsebenen teilweise sehr kompliziert Sprachebene

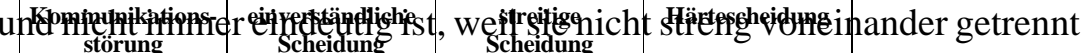
existieren, sojadern sick wechselscitgig beeinflussen und durchdringen, Sachinhaltsebengestand ein€eder Hauptschwierigkiten beim Ausfüllen der Fragebögen darin, die aufsetreterexommunikationsstörungera den entsprechenden

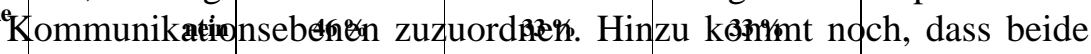

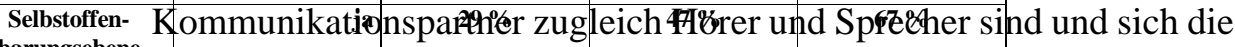
barungsebene

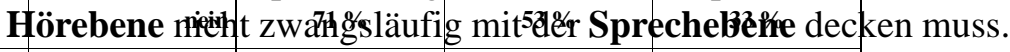

Appellebene $\quad$ Bei den Scheiduñ4s: menkomplex, der zu massiven Kommunikationsstörungen führt: das Nichtbekanntsein und/oder Nichtanerkennen des Zerrüttungsprinzips.

In elf Fällen war die Kommunikation zum Zerrüttungsprinzip gestört. Die Mandanten gingen immer noch vom Verschuldensprinzip aus, während der Gesetzgeber sich für das Zerrüttungsprinzip entschieden hat. So wurde immer wieder beim Anwalt argumentiert, dass es doch der Ehepartner sei, der sich einem neuen Partner oder einer neuen Partnerin zugewandt habe, und dass dieser Fakt im Scheidungsverfahren berücksichtigt werden müsse. So versuchte zum Beispiel ein Mandant perma- 
nent, die „Schuld“ seiner Frau am Scheitern der Ehe (sie hatte ihn und die Kinder verlassen) in den Mittelpunkt der Mandantengespräche zu rücken. Dadurch war zeitweilig keine Verständigung zwischen Anwalt und Mandanten mehr möglich, da Gespräche auf der Sachebene abgeblockt wurden. In einem anderen Fall versuchte eine Mandantin mittels rechtlicher Schritte, die Beziehung ihres Mannes zu einer anderen Frau zu unterbinden. Ihr war seitens des Anwaltes nur schwer vermittelbar, dass dieser Wunsch rechtlich nicht durchzusetzen ist.

Hier zeigt sich ein Problem, das das gesamte Scheidungsverfahren durchzieht: Die Ehepartner sind verletzt und die Stimmung zwischen ihnen ist emotional aufgeheizt; teilweise ist gar keine Verständigung mehr möglich und die Ehepartner kommunizieren nur noch über ihre Prozessbevollmächtigten miteinander. Die bereits bestehenden Differenzen werden auf die Kommunikation mit dem Anwalt übertragen. Die zerstrittenen Eheleute hören wechselseitig nur auf dem „Beziehungsohr“ und sind einer sachlichen Argumentation ihres Anwaltes nicht mehr zugänglich. Dieser Konflikt strahlt oftmals auch auf die anderen Kommunikationsebenen aus. Als Konsequenz werden auf der Selbstoffenbarungsebene entweder überhaupt keine expliziten Aussagen mehr getroffen, oder es herrschen im anderen Extremfall Minderwertigkeitsgefühle, Schuldkomplexe sowie ein fast pathologisches Unwertgefühl vor. Die Appellebene hingegen wird genutzt, um Vorwürfe an den Ehepartner zu formulieren und Schuldgefühle bei ihm hervorzurufen. Das Hören auf der Appellebene wird dagegen blockiert. Die Emotionalität der Gespräche geht einher mit einer Erwartungshaltung der Mandanten, dass bei der Erörterung der rechtlichen Folgen auch moralische Wertungen einbezogen werden und damit die „Gerechtigkeit“ wieder hergestellt wird. Weder der Anwalt noch der Richter kann dieser Erwartungshaltung entsprechen. Das führt bei dem oft sehr verletzten und gedemütigten Mandanten zu Unverständnis, Enttäuschung und in der Folge oft zu den eben dargestellten Kommunikationsstörungen. 


\section{4. $\mathrm{Zu}$ ausgewählten Scheidungsfolgen}

Sowohl das Sorgerecht ${ }^{5}$ als auch der Versorgungsausgleich müssen zwingend von Amts wegen geregelt werden. Dabei ist zu beachten, dass im Zusammenhang mit der elterlichen Sorge auch über das Aufenthaltbestimmungsrecht sowie das Umgangsrecht gesprochen werden muss.

Bezüglich des Versorgungsausgleichs sind die kommunikativen Störungen in der mündlichen Kommunikation zwischen Anwalt und Mandanten vergleichsweise gering. Zwar ist den wenigsten Mandanten der rechtssprachliche Terminus Versorgungsausgleich bekannt, diese Wissenslücke kann aber vom erfahrenen Anwalt schnell geschlossen werden. Verständnisprobleme sind also weniger an den Terminus geknüpft, sondern entstehen eher aus dem Unverständnis der Mandanten heraus, dass die Berechnung des Versorgungsausgleichs das Scheidungsverfahren erheblich verzögert. ${ }^{6}$ Dieses Unverständnis gipfelte in einem Fall in dem Vorwurf gegen den Anwalt, er unternehme nichts, um eine sofortige Scheidung durchzudrücken. Die vom Anwalt benannten formal-juristischen Hindernisse nach § 628 I Nr. 3 ZPO wurden dabei nur schwer akzeptiert. Es handelt sich bei dem eben angeführten Beispiel wiederum um eine gestörte Verständigung auf der Beziehungsebene, wobei der Vorwurf der Untätigkeit natürlich auch die Appellebene durchdringt, um ein Tätigwerden des Anwalts zu erreichen. Beim Thema Versorgungsausgleich tritt außerdem das Problem auf, dass emotional verletzte Mandanten die Notwendigkeit dieses Verfahrensschritts nicht akzeptieren und folglich auch sachlichen Argumenten nicht zugänglich sind.

Für zum Teil massiv auftretende Probleme ${ }^{7}$ beim Ausfüllen des Fragebogens zum Versorgungsausgleich - also bei der schriftlichen Kommunikation - gibt es mehrere Ursachen:

5 Mit dem Inkrafttreten des neuen KindRG wird seit 01.07.1998 über die elterliche Sorge nur noch auf Antrag eines Elternteils entschieden, ansonsten bleibt es bei der gemeinsamen Sorge für gemeinsame Kinder.

6 Eine Berechnungsdauer von ein bis anderthalb Jahren ist nach Aussage von Richtern und Anwälten keine Seltenheit.

7 Nach Aussage eines langjährigen Familienrichters haben ca. 20\% der Antragsteller bzw. Antragsgegner gravierende Schwierigkeiten beim Ausfüllen der Formulare. In etwa 20-30 \% der Fälle werden die Formulare überhaupt nicht ausgefüllt. 
1. Die einzureichenden Unterlagen zum Versorgungsausgleich sind sehr umfangreich und kompliziert. Dazu gehören ein vierseitiger Fragebogen mit zweiseitigen Erläuterungen zum Ausfüllen, ein vierseitiger Antrag auf Kontenklärung mit sechsseitiger Erläuterung sowie ein Formblatt zur Vorlage beim Rentenversicherungsträger. Diese Formulare müssen in doppelter Ausfertigung ausgefüllt werden. Des weiteren sind eine beglaubigte Kopie des Sozialversicherungsbuches, die eigene Geburtsurkunde, Geburtsurkunden der Kinder und das Schulabschlusszeugnis beizubringen. Allein die Quantität und Komplexität der geforderten Auskünfte führt zu Verständnisproblemen auf der Sachinhaltsebene und schreckt viele Antragsteller bzw. Antragsgegner im Scheidungsverfahren so ab, dass sie sich die Unterlagen gar nicht näher ansehen und die Aufforderung des Gerichts ignorieren, die geforderten Unterlagen binnen eines Monats vollständig ausgefüllt an das Familiengericht zurückzusenden. Dazu kommt eine jahrelang durch eigene Erfahrungen geprägte Erwartungshaltung, dass Behördenschreiben und amtliche Formulare grundsätzlich schwer oder gar nicht zu verstehen sind. Diese Erwartungshaltung wird durch umständliche Erläuterungen sowie die gehäufte (und zum Teil unnötige) Verwendung von Fachtermini in den oben genannten Formularen leider bestätigt.

2. In einigen Fällen führen auch finanzielle Überlegungen dazu, den Versorgungsausgleich zu blockieren, indem die notwendigen Informationen nicht gegeben werden. Die Vorstellung, die eigene Rente eventuell durch den Ausgleich der Rentenanwartschaften zu schmälern und dem Ex-Partner so zu einer höheren Rente zu verhelfen, führt zu einer Verweigerungshaltung, die auch auf die Kommunikation ausstrahlt.

3. Einige Mandanten weigern sich prinzipiell, die notwendigen Formulare auszufüllen mit der Begründung, dass sie die Ehescheidung nicht gewollt und zu diesem Schritt auch keinen Anlass geboten hätten. Hier zeigt sich wieder das Problem der emotionalen Betroffenheit und des Reagierens auf der Beziehungsebene, das eine kommunikative Blockierung nach sich zieht.

4. Ähnlich sieht es mit dem Personenkreis aus, der grundsätzlich amtliche Formulare ignoriert. Hier fallen Probleme auf unterschiedlichen Kommunikationsebenen zusammen, zum einen eine Überforderung 
auf der Sachinhaltsebene und zum anderen wieder Schwierigkeiten auf der Beziehungsebene aufgrund einer negativen Erwartungshaltung gegenüber staatlichen Behörden und Gerichten. Die totale Verweigerungshaltung verzögert das Scheidungsverfahren und zieht rechtliche Konsequenzen bis hin zur Verhängung einer Geldstrafe nach sich. Bevor es soweit kommt, greifen gute Anwälte vermittelnd ein und helfen ihren Mandanten beim Ausfüllen der Formulare, damit das Scheidungsverfahren nicht künstlich in die Länge gezogen wird. Dabei haben die Anwälte allerdings mit den gleichen Problemen und Vorbehalten wie unter 1.-4. dargestellt zu kämpfen.

\begin{tabular}{|l|r|c|c|}
\hline Sprachebene & Kommunikationsstörung & $\begin{array}{c}\text { Versorgungsaus- } \\
\text { gleich }\end{array}$ & Sorgerecht \\
\hline Sachinhaltsebene & ja & $33 \%$ & $55 \%$ \\
& nein & $67 \%$ & $45 \%$ \\
\hline Beziehungsebene & ja & $33 \%$ & $61 \%$ \\
Selbstoffenbarungs- & nein & $67 \%$ & $39 \%$ \\
ebene & ja & $21 \%$ & $71 \%$ \\
\hline Appellebene & nein & $79 \%$ & $29 \%$ \\
\hline
\end{tabular}

Tabelle 4

Wie Tabelle 4 veranschaulicht, treten die meisten Kommunikationsstörungen jedoch nicht beim Thema Versorgungsausgleich, sondern beim Streit um das Sorgerecht auf. Dieses Ergebnis überrascht nicht, denn beim Kampf um das gemeinsame Kind oder die gemeinsamen Kinder werden all jene Emotionen frei, die durch die Verletztheit der Scheidungsparteien in der partnerschaftlichen Beziehung hervorgerufen werden. Viele Ehepaare können im Falle einer Scheidung nicht mehr zwischen der Partnerebene und der Elternebene trennen. Alle negativen Empfindungen dem Partner gegenüber wie Enttäuschung, Wut und teilweise auch Hass brechen sich bei diesem Thema fast ungebremst Bahn, denn über das Sorgerecht kann Macht neu definiert und der „Gegner“, also der Ehepartner, in die Defensive gedrängt werden. Dies geschieht alles meist unter der Überschrift „Kindeswohl“, obwohl bei den aggressiv und unfair geführten 
Auseinandersetzungen um Sorge- und Umgangsrecht von vornherein ein Verlierer feststeht: das eigene Kind, das allein schon durch die Scheidung emotional stark belastet wird und nun zusätzlich durch das Hinund Hergerissensein zwischen Vater und Mutter leidet. Denn es ist noch immer Scheidungsalltag, dass um das Sorgerecht erbittert gestritten wird. Nur in $16 \%$ der empirisch erfassten Fälle wurde das gemeinsame Sorgerecht beantragt. In $84 \%$ der Fälle wurde das alleinige Sorgerecht entweder auf die Mutter oder auf den Vater übertragen.

Die Kommunikationsstörungen beim Thema Sorgerecht betreffen alle Kommunikationsebenen. Die geringsten Schwierigkeiten gibt es dabei auf der Sachinhaltsebene. Wie schon beim Versorgungsausgleich dargestellt, lassen sich terminologische Unklarheiten relativ schnell aufklären. Missverständnisse traten zum Beispiel bei der Unterscheidung zwischen alleinigem und gemeinsamen Sorgerecht sowie zwischen Aufenthaltbestimmungsrecht und Umgangsrecht auf. Diese Missverständnisse führten aber nicht zwangsläufig zu unüberwindbaren Kommunikationsschwierigkeiten. Die anwaltliche Beratung war im Beispielfall erfolgreich und führte im Ergebnis zu einer gütlichen Sorgerechtsvereinbarung.

In den Fällen, in denen das gemeinsame Sorgerecht beantragt wurde, gab es selten Kommunikationsstörungen. Das bestätigt die These, dass einvernehmliches Handeln der Scheidungsparteien Kommunikationsstörungen verhindern oder zumindest verringern kann. In Einzelfällen handelten die Eltern auch dann einvernehmlich, wenn ein Elternteil das alleinige Sorgerecht beantragt hatte. Hier dominierte offensichtlich die Einsicht der Eltern, für ihre Kinder eine möglichst optimale Lösung zu finden. Die Kinder wurden in den Entscheidungsprozeß einbezogen und ihre Wünsche wurden berücksichtigt. Beide Elternteile verzichteten auf Drohgebärden und Machtspiele.

Leider sind diese Beispiele Einzelfälle. Viel häufiger kommt es zu einem erbitterten Streit um die Kinder. Dabei steht - wie auch bei anderen Themen - eine gestörte Verständigung auf der Beziehungsebene einer von den Scheidungsparteien selbst verantworteten Regelung im Weg.

Weitere Scheidungsfolgen, über die im Rahmen einer Ehescheidung gesprochen und oft genug auch gestritten wird, sind der Ehegatten- und Kindesunterhalt, der Zugewinnausgleich, der Hausrat sowie die

Ehewohnung. Kommunikationsstörungen waren bei allen genannten Scheidungsfolgen vorhanden, allerdings schwankte ihre Häufigkeit nach 
der Brisanz der Themen. Die zum Teil vorhandene Massivität der Verständnisprobleme lässt sich ganz allgemein damit erklären, dass es vor allem um materielle Streitigkeiten geht, also um Geld und andere materielle Werte. Und beim Geld hört bekanntlich nicht erst bei einer Ehescheidung die Freundschaft auf. Niemand will gern von seinem Geld etwas abgeben, und schon gar nicht dem Ehepartner, der ihn oder sie vielleicht auch noch betrogen hat. So lautet sehr häufig die Argumentation beim Anwalt, wenn es um die Klärung der oben genannten Themen geht. In vielen Fällen wurde erbittert um jede Mark gestritten. Die Scheidungsparteien versuchten, wie vorher schon beim Streit um das Sorgerecht, den Ex-Partner zu bestrafen, indem sie ihn möglichst auch finanziell schädigten. Die meisten Kommunikationsstörungen traten dabei erwartungsgemäß bei der Frage nach Kindesunterhalt und Ehegattenunterhalt auf. Sie beeinflussten in unterschiedlicher Intensität die vier Kommunikationsebenen, wie Tabelle 5 zeigt.

\begin{tabular}{|c|c|c|c|c|c|c|}
\hline Sprachebene & $\begin{array}{l}\text { Kommunika- } \\
\text { tionsstörung }\end{array}$ & $\begin{array}{c}\text { Kindes- } \\
\text { unterhalt }\end{array}$ & $\begin{array}{c}\text { Ehegatten- } \\
\text { unterhalt }\end{array}$ & $\begin{array}{c}\text { Zugewinnaus- } \\
\text { gleich }\end{array}$ & Hausrat & $\begin{array}{c}\text { Ehe- } \\
\text { wohnung }\end{array}$ \\
\hline $\begin{array}{l}\text { Sachinhalts- } \\
\text { ebene }\end{array}$ & $\begin{array}{r}\text { ja } \\
\text { nein }\end{array}$ & $\begin{array}{l}46 \% \\
54 \%\end{array}$ & $\begin{array}{l}62 \% \\
38 \%\end{array}$ & $\begin{array}{l}52 \% \\
48 \%\end{array}$ & $\begin{array}{l}44 \% \\
56 \%\end{array}$ & $\begin{array}{l}\mathbf{5 0} \% \\
\mathbf{5 0} \%\end{array}$ \\
\hline $\begin{array}{l}\text { Beziehungs- } \\
\text { ebene }\end{array}$ & $\begin{array}{r}\text { ja } \\
\text { nein }\end{array}$ & $\begin{array}{l}54 \% \\
46 \%\end{array}$ & $\begin{array}{l}54 \% \\
46 \%\end{array}$ & $\begin{array}{l}43 \% \\
57 \%\end{array}$ & $\begin{array}{l}39 \% \\
61 \%\end{array}$ & $\begin{array}{l}40 \% \\
60 \%\end{array}$ \\
\hline $\begin{array}{l}\text { Selbstoffen- } \\
\text { barungsebene }\end{array}$ & $\begin{array}{r}\text { ja } \\
\text { nein }\end{array}$ & $\begin{array}{l}33 \% \\
67 \%\end{array}$ & $\begin{array}{l}42 \% \\
58 \%\end{array}$ & $\begin{array}{l}33 \% \\
67 \%\end{array}$ & $\begin{array}{l}22 \% \\
78 \%\end{array}$ & $\begin{array}{l}40 \% \\
60 \%\end{array}$ \\
\hline Appellebene & $\begin{array}{r}\text { ja } \\
\text { nein }\end{array}$ & $\begin{array}{l}62 \% \\
38 \%\end{array}$ & $\begin{array}{l}54 \% \\
46 \%\end{array}$ & $\begin{array}{l}43 \% \\
57 \%\end{array}$ & $\begin{array}{l}33 \% \\
67 \%\end{array}$ & $\begin{array}{l}30 \% \\
70 \%\end{array}$ \\
\hline
\end{tabular}

Tabelle 5

Die auftretenden Kommunikationsstörungen auf der Sachinhaltsebene wurden im allgemeinen nicht dadurch hervorgerufen, dass die Mandanten grundsätzlich eine Pflicht zur finanziellen Unterstützung ihrer Kinder verneinten. Vielmehr wurde die sachliche Akzeptanz der Unterhaltsforderungen dadurch untergraben, dass Probleme auf der Beziehungsebene wie auch schon beim Sorgerecht die notwendige Trennung von Partnerschafts- und Elternebene verhinderten. So warfen sich Scheidungsparteien in einem Fall wechselseitige Ausbeutung vor und verdrängten dabei, 
dass der Unterhalt für die Kinder bestimmt ist. Es gab allerdings auch Fälle, in denen seitens der Unterhaltsverpflichteten jegliche Unterhaltszahlungen verweigert wurden. Zur Begründung wurde von den Mandanten angegeben, dass ihre Partnerin an der Ehescheidung schuld sei, und sie deshalb nicht bereit wären, für die Folgen aufzukommen.

Bei der Berechnung von Unterhaltsleistungen wurde seitens einiger Mandanten nicht verstanden, dass der Unterhaltsverpflichtete einen Selbstbehalt hat, der bei Unterhaltsforderungen berücksichtigt wird und nicht unterschritten werden kann. Es handelt sich hierbei um eine typische Kommunikationsstörung auf der Sachinhaltsebene, die weniger durch Unkenntnis des Terminus Selbstbehalt, sondern mehr durch das Unverständnis des diesem Terminus zugrunde liegenden Rechtsverständnisses hervorgerufen wurde. So wollte eine Mandantin die Unterhaltsverpflichtung ihres Mannes nicht auf den Selbstbehalt begrenzen mit der Begründung, dass ihr dieser Selbstbehalt auch nicht zur Verfügung stünde. Diese Reaktion ist aus rein menschlicher Sicht sicher nicht unverständlich, doch sie verweist auf das eben genannte, tiefer liegende Problem: Unkenntnis oder Nichtakzeptanz des zugrunde liegenden Rechtssystems.

Eng mit der finanziellen Problematik verbunden ist auch das Thema Zugewinn. Die Kommunikationsstörungen waren allerdings wesentlich geringer als bei den Themen Kindes- und Ehegattenunterhalt. Das resultiert vor allem daraus, dass in mehreren Fällen Eheverträge existierten und somit kein Regelungsbedarf bestand. In einigen Fällen wurden sowohl das Problem des Zugewinns als auch anderer Scheidungsfolgen außergerichtlich erarbeitet und dem Gericht zur Protokollierung vorgelegt. Die meisten Kommunikationsstörungen traten indes auf der Sachinhaltsebene auf. Hierbei handelte es sich vorwiegend um echte terminologische Unsicherheiten den Terminus Zugewinn betreffend, da dieser einigen Mandanten nicht bekannt war. Daneben waren die schon bei anderen Themen analysierten Kommunikationsschwierigkeiten durch Vermischung der Sachinhalts- und der Beziehungsebene zu beobachten.

Zusammenfassend lässt sich sagen: Die geringsten Kommunikationsstörungen, die in der anwaltlichen Kommunikation auftreten, liegen auf der Sachinhaltsebene. Sie sind sowohl quantitativ als auch qualitativ einzugrenzen, zumeist rückführbar auf die schwierige juristische Terminologie und am wenigsten störend für eine erfolgreiche Kommunikation. Die meisten Probleme treten auf der Beziehungsebene auf. Ausführliche 
Begründungen, warum das so ist, wurden bereits umfassend dargelegt. Am schwierigsten ist die Ursachenforschung für Kommunikationsstörungen weiterhin auf der Selbstoffenbarungsebene. Viele Mandanten sind nicht bereit, freiwillig etwas von sich preiszugeben und wenn, dann meist nur in der Absicht, sich selbst im besten Licht darzustellen und den Ehepartner zum jeweilig erörterten Thema zu diffamieren. Natürlich geben die Mandanten dadurch unbeabsichtigt etwas von sich preis. Der geschulte Anwalt wird nun versuchen, die hinter Anschuldigungen, Enttäuschungen und verletzten Gefühlen liegenden ,wahren“ Beweggründe und Interessen seines Mandanten herauszufinden. Das ist allerdings sehr schwer und gelingt oft nur teilweise. Einige Mandanten geben auch deshalb wenig oder nichts freiwillig von sich preis, weil sie vermuten, dass es gegen sie verwendet wird. Das ist entweder dann der Fall, wenn es wirklich etwas zu verbergen gibt (zum Beispiel Alkoholsucht oder psychische Krankheiten, die eine Sorgerechtsentscheidung des Gerichts beeinflussen könnten), oder wenn das Selbstwertgefühl des Mandanten sehr gering ist und er vermutet, dass ihm sowieso nicht geglaubt wird oder seine Aussage unwichtig ist. Diese Mandanten haben ein gestörtes Vertrauensverhältnis zu ihrem Anwalt, weil sie ihn nicht als Partner und Interessenvertreter verstehen und ihm mit Skepsis begegnen.

Auf der Appellebene sind verschiedene „Verhaltensmuster“ zu beobachten. Grundsätzlich sind auch hier Vermischungen mit der Beziehungsebene vorhanden. So werden häufig appellartige Vorwürfe an den Ehepartner gerichtet, um ihn (oder sie) zur Unterhaltszahlung zu bewegen. Es finden sich aber auch resignative Haltungen mit Begründungen wie: Es hat sowieso alles keinen Sinn. Ich kann die Situation nicht ändern und beeinflussen. Hier zeigt sich einmal mehr, wie vorgeprägte Erwartungshaltungen bezüglich der Allmacht des Gerichts dazu führen können, die Eigenverantwortlichkeit der Scheidungsparteien in den Hintergrund zu drängen und die Passivität der Mandanten zu fördern. Die Appellebene wird aber auch von einigen Mandanten dazu genutzt, dem eigenen Anwalt Vorwürfe zu machen, dass er die Interessenvertretung nicht wahrnimmt und das Verfahren verzögert. Ursachen für diese Reaktion sind zum einen die mangelnde Kenntnis der Verfahrensabläufe und Zuständigkeiten und zum anderen die Ignoranz der Verfahrensabläufe und Zuständigkeiten. 


\subsection{Die Kommunikation mit dem Anwalt aus Sicht der Mandanten}

In den vorangegangenen Kapiteln wurden Kommunikationsstörungen schwerpunktmäßig aus der Sicht der Fachleute betrachtet. Um auftretende Verständigungsschwierigkeiten umfassend beurteilen zu können, muss natürlich auch die Perspektive der Laien analysiert werden. Allerdings ergeben sich in diesem Bereich bei der empirischen Datengewinnung schwerwiegende objektive Hindernisse, die eine repräsentative Untersuchung verhindern. Zum einen sind nur sehr wenige Betroffene bereit, über ihre Erfahrungen zu sprechen und ihr Kommunikationsverhalten einzuschätzen. Zum anderen sind Scheidungsverhandlungen nicht öffentlich, so dass eine Einschätzung der gerichtlichen Kommunikation in dieser Weise eigentlich nicht erfolgen kann. Trotzdem soll auf die Laienperspektive nicht vollständig verzichtet werden, obwohl die nachfolgenden Erkenntnisse und Aussagen nur punktuell erfolgen können und einer weitergehenden Untersuchung bedürfen. ${ }^{8}$ Interessant sind insbesondere die Aussagen von geschiedenen oder in Scheidung lebenden Personen, da sie Aussagen der Fachleute in weiten Teilen bestätigen und ergänzen.

Für den Bereich der anwaltlichen Kommunikation stellt sich die Situation aus Sicht der Betroffenen wie folgt dar: In der Tat ist eine Hemmschwelle vorhanden, im Falle einer drohenden Scheidung einen Anwalt aufzusuchen. Da aber zumindest der Antragsteller anwaltlich vertreten sein muss, ist naturgemäß die Hemmschwelle des Partners, der die Scheidung einreichen will (Antragsteller), niedriger als die des Partners, der auf den Scheidungsantrag reagieren muss (Antragsgegner). Trotzdem kümmert sich in der Mehrzahl der Fälle auch der Antragsgegner um anwaltliche Unterstützung, denn die Angst, bei der gerichtlichen Verhandlung die eigene Rechtsposition zu schwächen, ist größer als die Angst vor dem Gang zum Anwalt. Die Bedenken, einen Anwalt zu Rate zu ziehen, bestehen dabei einmal in finanzieller Hinsicht und zum anderen in dem Unbehagen, einem Fremden sehr private Details anzuvertrauen und sich ihm quasi auszuliefern. Hinzu kommt die Rollenerwartung: Der

8 Die nachfolgenden Erkenntnisse stützen sich auf Befragungen und Interviews von geschiedenen oder in Scheidung liegenden Männern und Frauen sowie auf die Teilnahme der Autorin an mehreren gerichtlichen Scheidungsverhandlungen. 
Anwalt ist Fachmann für rechtliche Belange und ist Spezialist auf dem für den Laien oft verwirrenden Gebiet des Verfahrensrechts. Dieses Vorwissen ist oft verbunden mit einem Gefühl der eigenen Unterlegenheit und Unwissenheit, das Unsicherheit im eigenen Kommunikationsverhalten nach sich zieht. Der Betroffene sieht in dem Anwalt den fachlich Überlegenen und vertraut ihm. Gerade aber das Gefühl der eigenen Unterlegenheit kann auch Misstrauen hervorrufen. Betroffene äußerten dabei die Befürchtung, dass der Anwalt ihre Unkenntnis ausnutzen könnte und nur auf ihre Kosten Geld verdienen wolle. Weiterhin wird vom Anwalt erwartet, dass er wie ein Fachmann auftritt - also vom Auftreten her sehr selbstbewusst und vertraut mit der entsprechenden Fachterminologie. Auf der anderen Seite aber erwarten die Betroffenen in der Kommunikation, dass der Anwalt kein „Fachchinesisch“ spricht. Die Verständigung wird auch dadurch erschwert, dass Mandanten davon ausgehen, dass der Anwalt ihre Gesamtsituation, die emotional oft als sehr schwierig empfunden wird, würdigt, und nicht nur die für ihn rechtlich relevanten Aspekte berücksichtigt. Wird der Anwalt diesem Anspruch nicht gerecht, führt dies häufig zu Kommunikationsschwierigkeiten, die oft in dem Vorwurf münden: Mein Anwalt tut nichts für mich und ist unfähig.

Auf das Problem des dem Ehescheidungsrecht zugrunde liegenden Zerrüttungsprinzips hin angesprochen, argumentierten die Betroffenen wie folgt: Die Tatsache, dass das bundesdeutsche Scheidungsrecht auf dem Zerrüttungsprinzip basiert, ist vielen Betroffenen nicht bekannt. Die Grundzüge dieses Prinzips werden aber seitens der Anwälte allgemeinverständlich und umfassend erklärt. Das Problem liegt also nicht in der Unkenntnis oder in der Kompliziertheit dieses Rechtsprinzips sondern in der mangelnden Akzeptanz. Für viele Betroffene ist völlig unverständlich, dass die Frage nach den Ursachen (oder nach dem Verursacher bzw. der Verursacherin) für das Scheitern der Ehe im Scheidungsverfahren und damit für die Scheidungsfolgen keine Rolle spielt. Sie können und wollen nicht akzeptieren, dass die Kategorien persönlicher Schuld und Verantwortung für die rechtliche Beurteilung unerheblich sind. Dieses Beispiel bestätigt folglich die empirisch getroffene Aussage, dass die Kommunikationsstörungen zwischen Anwalt und Mandanten schwerpunktmäßig auf der Beziehungsebene entstehen und erst an zweiter Stelle durch Verständigungsschwierigkeiten auf der Sachinhaltsebene bedingt sind. 


\section{Zusammenfassung und Ausblick}

Die Zahl der Ehescheidungen in Deutschland hat einen neuen Höchststand erreicht. Nach Angaben des Statistischen Bundesamtes in Wiesbaden ließen sich im Jahr 2000 194.410 Paare scheiden.

Diese alarmierenden Zahlen sprechen eine deutliche Sprache. Nie zuvor waren in Deutschland so viele Menschen mit dem Thema „Ehescheidung" konfrontiert. Nach den aktuellen Angaben sind von 1000 Ehen zehn zerbrochen. Was die Statistik verschweigt, sind die menschlichen Schicksale, die Verzweiflung, Enttäuschung, Wut und Unsicherheit der Betroffenen, die sich hinter diesen Zahlen verbergen. Doch diese Emotionen und Empfindungen sind immer gegenwärtig - beim Gespräch mit dem Scheidungsanwalt, bei der mündlichen Gerichtsverhandlung. Und sie führen zu Problemen, auch in der Kommunika-tion.

Die empirische Untersuchung zeigt, dass viele der auftretenden Kommunikationsstörungen, sei es zwischen Anwalt und Mandanten oder zwischen Richter und Parteien, nicht allein durch terminologische Schwierigkeiten auf der Sachinhaltsebene, sondern durch Missverstehen oder sogar Nichtverstehen auf der Beziehungsebene hervorgerufen werden. Weitere Verständigungsprobleme, die eng damit verbunden sind, treten auf der Selbstoffenbarungs- und auf der Appellebene auf. Durch diese Untersuchungsergebnisse wurde nachgewiesen, dass Kommunikationsstörungen in der fachsprachlichen Vermittlung an den Laien nur zum Teil durch die sprachlichen Besonderheiten der Fachsprache des Rechts hervorgerufen werden. Der andere Teil setzt sich aus Kommunikationsstörungen zusammen, die vor allem durch kontextuelle sprachliche und außersprachliche Bedingungen der Kommunikation wie zum Beispiel Rollenerwartungen, persönliche Erfahrungen und Interpretationen der Kommunikationsteilnehmer, Angst vor finanziellen Einbußen beim Gang zum Anwalt sowie Unkenntnis der Grundlagen des deutschen Rechtssystems und prozessualer Erfordernisse erzeugt werden.

Mögliche Lösungsansätze sind so vielfältig wie die aufgetretenen und untersuchten Kommunikationsschwierigkeiten. Auf sprachlicher Ebene ist dabei an erster Stelle die Einhaltung der kommunikationskonstituierenden Prinzipien (Heringer 1977: 173) zu nennen. Diese Forderung richtet sich sowohl an den Fachmann als auch an den Laien. Weiterhin muss sich insbesondere der Anwalt der Bedeutung seiner Vermittlerposition im Kommunikationsprozess bewusst sein, ohne dabei zu vergessen, 
dass jeder Mandant für seinen speziellen Fall der ,eigentliche“ Fachmann ist. Außergerichtliche Konfliktlösungsmethoden wie das Mediationsverfahren, deren Elemente sich auch Richter in der mündlichen Verhandlung zu eigen machen sollten, können dabei unterstützend wirken. Weitergehende, nicht allein sprachlich begründete Lösungsansätze sollen bereits im Vorfeld möglicher rechtlicher Streitigkeiten versuchen, durch die gezielte Erweiterung des Rechtskundeunterrichts an Schulen und durch populärwissenschaftliche Sendungen, Kenntnisdefizite über das deutsche Rechtssystem abzubauen, und somit vorbeugend zu wirken. Auch die Möglichkeit einer dem Gerichtsprozess vorgelagerten prozessualen Beratung, die die anwaltliche Beratung vorbereiten und unterstützen kann, sollte ernsthaft in Betracht gezogen werden.

Alle diese Überlegungen sind dabei letztendlich auch ein Beitrag zur Sprachkultur und Sprachpflege. Dabei bezieht sich Sprachkultur nicht nur auf den Zustand des Sprachsystems und seine „Eignung“ für die Befriedigung der kommunikativen und kognitiven Bedürfnisse der Sprachteilhaber und ist auch nicht begrenzt auf die - wie auch immer verstandene - Qualität der Textproduktion. Wenn man sie auffasst als „das Niveau des Gebrauchs, den die Kommunikationsgemeinschaft von der Sprache macht, dann erscheint sie als Qualität sprachlich-kommunikativen Handelns, als „Realisierung menschlicher Produktivität“, und man könnte dann eher von Kommunikationskultur, der Kultur kommunikativen Verhaltens sprechen. Da Kommunikation ein Prozess wechselseitiger Produktion und Rezeption von Kommunikationspartnern ist, hat die rezeptive Fähigkeit nicht weniger Gewicht als die produktive, und Förderung oder Verbesserung der Sprachkultur schließt beide ein. " (Fleischer 1993: 289).

Für eine erfolgreiche Kommunikation sind also beide Kommunikationspartner von Bedeutung, ob als Rezipient oder als Produzent; ob als Fachmann oder als Laie. Und diese Erkenntnis lässt sich auch auf die interdisziplinäre Zusammenarbeit von Germanisten und Juristen übertragen. Beide brauchen einander und können sich sinnvoll ergänzen. Noch steht die eingangs erwähnte Gartentür im sie beide trennenden Gartenzaun nur einen Spalt breit offen. Germanisten und Juristen sollten Sorge tragen, dass diese Tür nicht wieder zufällt und bald weit und einladend geöffnet wird. 


\section{Literaturverzeichnis}

Eckardt, Birgit (2000). Fachsprache als Kommunikationsbarriere? Verständigungsprobleme zwischen Juristen und Laien. Wiesbaden: Deutscher Universitäts-Verlag.

Fleischer, Wolfgang; Hartung, Wolfdietrich; Schild, Joachim; Suchsland, Peter (1993). Stilistik der deutschen Gegenwartssprache. Frankfurt am Main, Berlin, Bern, New York, Paris, Wien: Peter Lang.

Fluck, Hans-Rüdiger (1996). Fachsprachen. Einführung und Bibliographie. Tübingen: Francke Verlag GmbH 5. überarb. und erweiterte Aufl.

Hattenhauer, Hans (1995). Denkfehler zeigen sich in Stilfehlern. Über Juristen- und Allgemeinsprache. In Frankfurter Allgemeine Zeitung 286 vom 08.12.1995. 17.

Heringer, Klaus-Jürgen u.a. (1977). Einführung in die praktische Semantik. Heidelberg: Quelle und Meyer 1.Aufl.

Löffler, Heinrich (1994). Germanistische Soziolinguistik. Berlin: Schmidt 2. überarb. Aufl.

Schulz von Thun, Friedemann (1981). Miteinander reden 1. Störungen und Klärungen. Allgemeine Psychologie der Kommunikation. Reinbek bei Hamburg: Rowohlt.

Selting; Margret (1987). Verständigungsprobleme. Eine empirische Untersuchung am Beispiel der Bürger-Verwaltungs-Kommunikation. Tübingen: Niemeyer.

Verlierer sind die Männer (1997). In Der Spiegel 47 vom 17.11.1997: Hamburg. 